\title{
Il pensiero pedagogico italiano nei manuali polacchi di storia dell'educazione
}

\author{
Italian education and pedagogical thought in Polish \\ textbooks on history of education
}

\begin{abstract}
This paper describes the popularization of the Italian pedagogical thought in Polish textbooks about the history of education in general. It has shown that the authors of the textbooks gave a lot of information about the Italian school system and education and presented profiles of outstanding Italian educationalists, e.g. St John Bosco or Maria Montessori. This paper indicates that in the past centuries Poles were open to Italian intellectual currents, academic trips to the Italian Peninsula, and education at local universities. Polish textbooks about the history of education were popular (in the 20th c.) and still are an important source of knowledge about the history of Italian education. For the purposes of this paper, the contents of the most representative and popular textbooks have been analysed.
\end{abstract}

\section{Keywords}

History of education, pedagogical thought, Italian school system, popularization of knowledge.

Il patrimonio culturale italiano ha da sempre suscitato un vivo interesse nei Polacchi e il processo della sua ricezione, costante nei secoli, si è tuttavia intensificato in alcuni periodi storici. I Polacchi desideravano conoscere opere d'arte, 
vita scientifica e vicende storiche della nazione italiana, in quanto affascinati dalle opere dei letterati della penisola italica. Un ruolo di spicco per la divulgazione della cultura italiana svolsero i numerosi viaggiatori polacchi che in Italia, ${ }^{1}$ oltre ad esplorare le risorse delle biblioteche italiane in cerca di tracce della presenza scientifica polacca, colsero l'occasione per familiarizzare con il patrimonio artistico italiano. Józef Kremer, detto il padre dellestetica polacca, nel suo "Viaggio in Italia" constata che in terra italiana "il pensiero del viaggiatore è pervaso dal soffio storico della civiltà. Infatti così come ogni popolo nei duri confronti con la storia, nelle crudeli battaglie, riportando vittoriosi stendardi si cingeva la fronte di corone d'alloro e ben felice deponeva le sue corone e stendardi nel tempio della divinità adorata, allo stesso modo l'Italia è considerata un sacrario per la storia universale del genere umano, per la sua gloria e i suoi meriti. Poiché quelle verità che nei millenni la storia aveva raggiunto, quelle verità - che definirei potenze secolari - che con tanto sangue e con tanta pena liberò dai recessi dell'animo umano, sono legate con la penisola italiana in una alleanza diretta o indiretta, e giustamente in questa terra eletta la storia lasciò i segni dei suoi trionfi”."

I Polacchi non solo viaggiavano in Italia ma attingevano anche alle opere di autori italiani volendo conoscere la pedagogia e il sistema organizzativo della didattica locale. Il sistema pedagogico italiano, per il suo carattere autonomo, sotto molti aspetti si differenziava da quello polacco e ciò fu uno dei fattori che determinarono la curiosità degli studiosi polacchi di storia dell'istruzione e delleducazione. Gran parte di loro esplorò a fondo il bagaglio storico culturale dell'Italia per tentare poi di presentare nei manuali di storia dell'educazione gli strumenti e le modalità di cui si serviva la società italiana per educare le giovani generazioni. Va sottolineato che i Polacchi recepivano in modo creativo non solo le idee dei pedagogisti italiani ma anche quelle dei rappresentanti di altri paesi come Francia, Inghilterra, Germania. ${ }^{3}$

Nei lavori scientifici attuali che riguardano i legami culturali italiano-polacchi mancano ancora libri ed articoli esaurienti che illustrino lo sviluppo dell'interesse polacco nei confronti del pensiero educativo e pedagogico italiano. Vi è infatti

${ }^{1}$ G. Maver, Podróze polskich pisarzy do Włoch, Rzym 1946, p. 9.

${ }^{2}$ J. Kremer, Podróż do Włoch, vol. 1. Droga z Krakowa do Triestu. Opisanie Wenecji, in: Dzieła Józefa Kremera, vol. VI, Warszawa 1978, p. 17.

${ }^{3}$ J. Dybiec, Recepcja idei pedagogicznych $w$ Polsce, in: Recepcja w Polsce nowych kierunków i teorii naukowych, a cura di A. Strzałkowski, Kraków 2001, pp. 195 e sc. 
la necessità di intraprendere studi in questo ambito. Il presente articolo ha lo scopo di mostrare in che modo nei manuali polacchi di storia delleducazione gli autori descrivono e valutano il pensiero educativo italiano. Ha senso interessarsi di questo argomento. I manuali universitari di storia dell'educazione sono per gli studenti degli orientamenti pedagogici la fonte primaria di informazioni sulla storia del sistema scolastico nei paesi esteri, e sullo sviluppo della teoria e della pratica pedagogica. Obiettivo dell'articolo sono le caratteristiche generali delle descrizioni della pedagogia italiana sulle pagine dei manuali polacchi di storia dell'educazione. È importante indicare quali, tra i pedagogisti italiani, hanno destato maggiore interesse in Polonia, e quali loro opinioni e risultati sono stati maggiormente apprezzati. Ė anche importante segnalare le differenze tra le opinioni dei singoli autori sul ruolo del sistema scolastico e del pensiero educativo italiano nella storia del sistema scolastico europeo.

\section{Caratteristiche generali dei manuali di storia dell'educazione}

In Polonia, la storia dell'educazione come disciplina scientifica autonoma nasce nel XIX secolo ${ }^{4}$ per effetto indubbio dello sviluppo delle scienze storiche e dellattenzione che gli studiosi del passato rivolgono alle scienze pedagogiche. Il primo Polacco che tenta di offrire un panorama sintetico della storia dell'istruzione e del pensiero pedagogico è Bronisław Ferdynand Trentowski, illustre pedagogista polacco dell'epoca del Romanticismo. Nella sua opera Chowanna, czyli system pedagogiki narodowej jako umiejętności wychowania, nauki i oświaty, słowem wykształcenia naszej młodzieży (vol. 1-2, Posen 1842) (L'arte di crescere ovvero il sistema di pedagogia nazionale come scienza delleducazione, istruzione ed insegnamento, in poche parole di formazione della nostra gioventù) dedica una delle parti del suo libro (epica) alle riflessioni sulleducazione attraverso i secoli.

Un crescente interesse verso la storia dell'educazione si fa notare nella seconda metà del secolo XIX. ${ }^{5}$ Lurgenza di scrivere compendi in materia derivava fra l'altro dal fatto che nei programmi formativi per gli insegnanti si raccomandava di insegnare la storia dell'educazione. Purtroppo mancavano manuali dai quali i futuri insegnanti potessero attingere per soddisfare

${ }^{4}$ H. Barycz, Rozwój historii oświaty, wychowania i kultury w Polsce, Kraków 1949.

${ }^{5}$ C. Majorek, System kształcenia nauczycieli szkół ludowych w Galicji doby autonomicznej (1971-1914). Wrocław 1971. 
la necessità di aggiornamento. Un primo tentativo di fornire un quadro sintetico della materia lo fece Antoni Łuczkiewicz, direttore dell'Istituto Statale Maschile di Magistero di Leopoli. Tuttavia il suo lavoro Szkolnictwo na podstawie historycznego rozwoju i zasad wychowania podług planu przepisanego dla seminariów nauczycielskich (Il sistema scolastico sulla base del suo sviluppo storico e dei suoi principi educativi alla luce del programma dinsegnamento previsto per gli istituti magistrali) pubblicato a Leopoli nel 1872 non era in manuale nel senso stretto del termine. Lo era invece un’opera scritta da Władyslaw Seredyński (Tarnów, 1891) Rys dziejów wychowania w biografiach i szkicach ze szczególnym uwzględnieniem szkoły ludowej $w$ Austrii i w Polsce dla użytku seminariów nauczycielskich polskich (Quadro storico delleducazione nelle biografie e nella saggistica, con particolare attenzione per la scuola popolare in Austria e Polonia, per l'uso degli istituti magistrali polacchi), un autore, che vantando una esperienza pedagogica pluriennale, aveva tutti i requisiti necessari per scrivere tale compendio. ${ }^{6}$

Nel secolo XX l'offerta editoriale polacca di manuali di storia dell'educazione diventa più ricca ${ }^{7}$ agli inizi della prima decade del secolo viene pubblicata Historia pedagogii dla użytku seminariów nauczycielskich (La Storia della Pedagogia ad uso degli Istituti magistrali) scritta da Franciszek Majchrowicz (Drohobycz, 1901). Lautore nel suo studio opera una distinzione fra la storia della pedagogica teorica e quella pratica, propone unanalisi critica delle idee riguardanti gli obiettivi dell'educazione e dei mezzi di realizzazione, offre infine un quadro storico delle istituzioni scolastiche. Sempre nel periodo ante bellico viene pubblicato a cura di Franciszek Bizoń un altro valido lavoro ${ }^{8}$ Historia wychowania ze źródeł czerpana. Podręcznik pedagogiczny dla użytku seminariów nauczycielskich, nauczycieli i ludzi zajmujących się wychowaniem (La storia delleducazione attinta da fonti scritte. Manuale di pedagogia ad uso degli istituti magistrali, degli insegnanti e di altri che si occupano dell'educazione). Il libro è impostato come unantologia di scritti pedagogici polacchi e stranieri.

${ }^{6}$ A. Meissner, Polskie podręczniki do historii wychowania, "Rozprawy z Dziejów Oświaty", vol. 32 (1989), p. 157.

7 S. I. Możdżeń, Praca naukowa historyka oświaty, Sandomierz 1999.

${ }^{8} \mathrm{Da}$ citare anche altri lavori che trattano la storia delleducazione, fra cui: M. Bielska, Historia pedagogii, Lwów 1890; K. Króliński, Zwięzły podręcznik historii pedagogii, Lwów 1907; W. Gadowski, Z historii pedagogii. Czasy od początku chrześcijaństwa do końca średniowiecza, Kraków 1889. 
Gli studi e le ricerche storiche nel campo della pedagogia conobbero uno sviluppo dinamico negli anni 1918-1939 ${ }^{9}$ grazie alla riconquista dell'indipendenza da parte della Polonia e alla fioritura dell'insegnamento universitario e dell'intensificarsi degli studi di ricerca in generale, e nella fattispecie, di quelli riguardanti levoluzione del pensiero pedagogico. Una posizione di spicco in quest'ambito spetta a Stanisław Kot, illustre umanista polacco, professore dell'Università Jagellonica di Cracovia, membro dell'Accademia Italiana delle Scienze. ${ }^{10}$ Fu proprio lui a costatare che in Polonia la storia dell'educazione era una delle scienze più trascurate. Le sue idee pionieristiche riguardanti la storia divergevano da quelle comunemente dominanti. Riteneva infatti la storia delleducazione una parte integrante della storia della cultura, intesa nel senso largo del termine, e perciò nei suoi lavori dedicati alla storia del pensiero pedagogico si riferiva frequentemente agli eventi culturali dell'epoca e in questa prospettiva cercava di analizzare quelli pedagogici, in rapporto alla situazione sociale, economica e culturale. ${ }^{11}$ Il contenuto del manuale di storia dell'educazione creato da S. Kot supera di gran lunga tutti gli altri compendi polacchi della disciplina. La prima edizione della sua Storia delleducazione risale al 1924, la seconda, in due volumi, viene pubblicata a Leopoli nel 1934. L'autore, convinto che nelleducazione pedagogica non si può far a meno di fonti storiche, pubblicò negli anni 1929-1930 due volumi di Fonti per la storia delleducazione, unopera a lungo fondamentale per lo studio della storia delleducazione. Dopo il 1956, indebolito il regime stalinista, ogni tentativo di ristamparla incontrò l'opposizione delle autorità governative che la ritenevano metodologicamente non conforme all'ideologia marxista.

Negli anni 1945-1989 la pressione ideologica comunista ostacolò con successo lo studio scientifico del pensiero pedagogico. ${ }^{12}$ In Polonia, paese che dopo la seconda guerra mondiale si era trovato nella sfera sovietica di influenza,

9 Fra i compendi storici di minor valore troviamo: A. Rzepecki, Skrót historii wychowania, Lwów 1921; J.S. Blaike, Historia wychowania. Repetytorium przedegzaminowe, Lwów 1925; E. Sokolowski, Skrót historii pedagogiki wedlug najnowszych podręczników szkolnych, Warszawa 1927.

${ }^{10}$ H. Barycz, Stanislaw Kot (1885-1975) - historyk polskiego Odrodzenia i Reformacji, "Biulletyn Biblioteki Jagielońskiej”, vol. 28 (1978), nn. 1-2, pp. 5-22; J. Dybiec, Stanislaw Kot (1885-1975), in: Złota księga Wydzialu Historycznego Uniwersytetu Jagiellonskiego, a cura di J. Dybiec, Kraków 2000, pp. 260-270.

11 S. Kot, Historia wychowania. Zarys podręcznikowy, Kraków 1924, p. 7.

12 S. Michalski, Miejsce historii wychowania w systemie nauk pedagogicznych, „Prace Komisji Pedagogiki i Psychologii Bydgoskiego Towarzystwa Naukowego”, Bydgoszcz 1981, p. 9. 
la censura eliminando alcuni dati riportati dalle pubblicazioni scientifiche rendeva impossibile l'oggettività della presentazione storica del lascito culturale delle generazioni passate. Il compito di scrivere un nuovo manuale viene assunto da Stefan Wołoszyn e nel 1964 nelle mani dei lettori giunse il suo Dzieje wychowania i myśli pedagogicznej w zarysie (Compendio di storia delleducazione e del pensiero pedagogico). Lautore, partendo dai presupposti della storiografia marxista, voleva inquadrare la storia dell'educazione "in rapporto allo sviluppo delle forze produttive e delle fasi della lotta di classe, con particolare riguardo a come nel campo della pubblica istruzione e delleducazione si riflettono gli obiettivi ideologici delle classi oppresse e la loro lotta per il progresso". ${ }^{13}$ Il manuale di Wołoszyn suscitò aspre critiche di numerosi recensori per la sua impostazione delle problematiche e per la mancata riflessione sulla storia della istruzione pubblica e dell'educazione.

Un coro di critiche più diversificate incontrò un altro compendio di storia dell'educazione, pubblicato negli anni 6o. del secolo scorso, Historia wychowania (La storia delleducazione) a cura di Łukasz Kurdybacha (voll.1-2, Varsavia 1965-1967) con la partecipazione di storici polacchi, sovietici e tedeschi orientali specializzati in varie epoche. Nel 1980 escono due volumi della Storia delleducazione - secolo XX (Historia wychowania wiek XX) a cura di Józef Miąso, alcune parti dei quali raccolgono recensioni positive. Negli anni 1945-1989, accanto ai sopracitati manuali basilari, vengono pubblicate dispense universitarie e brevi studi destinati a studenti dell'indirizzo pedagogico-didattico. ${ }^{14}$

Con la riconquista, nel 1989, della piena sovranità in Polonia si riapre il discorso sulla necessità di scrivere nuovi manuali di storia delleducazione che presentino in modo completo ed esauriente l'evoluzione del pensiero pedagogico. ${ }^{15}$ Tra i manuali pubblicati negli ultimi due decenni ${ }^{16}$ senza alcun dubbio merita la nostra attenzione il lavoro in tre volumi scritto da Stefan Możdżeń, Historia

13 S. Wołoszyn, Dzieje wychowania i myśli pedagogicznej w zarysie, Warszawa 1964, p. 15.

${ }^{14}$ Vedi anche: E. Podgórska, K. Kotłowski, Materiały do historii wychowania, Łódź 1956; P. Guz, Zarys historii wychowania, parte 1. Opole 1961; J. Szelejewski, Historia wychowania w zarysie, Poznań 1962; S. Rutkowski, W. Lisowski, Z. Doroba, Wybrane zagadnienia z dziejów wychowania $w$ Polsce, Warszawa 1970; Z. Marciniak, Zarys historii wychowania, Warszawa 1978; J. Krasuski, Historia wychowania, Warszawa 1985.

15 Historia wychowania $w$ XX w. Dorobek i perspektywy, Kielce 1998, p. 57.

16 Vedi anche: Historia wychowania. Skrypt dla studentów studiów dziennych i zaocznych, a cura di J. Hellwig, Poznań 1994; K. Bartnicka, I. Szybiak, Zarys historii wychowania, Warszawa 2001; M. Krajewski, Dzieje wychowania i doktryn pedagogicznych, Płock 2003. 
wychowania (Storia delleducazione), pubblicato a Kielce negli anni 2000-2005. L'autore - professore presso l'Akademia Swiętokrzyska di Kielce, la Scuola Superiore di Lettere e Scienze Naturali di Sandomierz e presso la sede distaccata dell'Università Cattolica di Lublino a Stalowa Wola - offre un quadro riassuntivo di tutte le epoche storiche trattando in modo innovativo certe tematiche finora mai toccate (fra cui l'educazione nella cultura mediorientale, bizantina, islamica, la pedagogia cristiana di San Paolo, dei Padri e dei Dottori della Chiesa).

Nel 2004, su iniziativa della Casa Editrice WAM e della Scuola Universitaria di Filosofia e Pedagogia "Ignatianum" di Cracovia, viene pubblicata Historia wychowania. Do Wielkiej Rewolucji Francuskiej (Storia delleducazione. Fino alla Grande Rivoluzione Francese) di Stanisław Litak. Il libro è basato su un pluriennale ciclo di conferenze tenute dall'autore presso l'Istituto di Storia e l'Istituto di Pedagogia dell'Università Cattolica di Lublino. Nel 2005, lo stesso tandem editoriale pubblica Historia wychowania. Wiek XIX i XX (Storia delleducazione. Secoli XIX e XX) scritta da Jan Draus e Ryszard Terlecki. Il manuale pone in rilievo il legame che secondo gli autori esiste fra la dimensione politica e l'educazione. Draus e Terlecki rivelano l'influenza esercitata nei secoli da ideologie totalitarie, in fattispecie da quella comunista, su concetti pedagogici ed istituzioni educative. ${ }^{17}$

I manuali polacchi di storia dell'educazione dal XIX secolo fino a nostri giorni forniscono un ricco bagaglio concernente la storia dell'educazione e della pedagogia italiana. Va segnalato però che gli autori dedicano maggiore spazio ad alcune problematiche a scapito di altre.

\section{Il pensiero pedagogico italiano dal Medioevo al Rinascimento}

Di particolare, e si direbbe costante, interesse per gli autori polacchi di manuali di storia dell'educazione è il sistema istruttivo italiano nel Medioevo, specialmente le problematiche riguardanti la nascita delle università. Trovava tutti d'accordo l'opinione che le università fossero sorte come risposta al crescente desiderio di sapere anche da parte delle nuove generazioni. ${ }^{18}$ I compendi

\footnotetext{
17 J. Draus, R. Terlecki, Historia wychowania. Wiek XIX i XX, Kraków 2005, p. 9.

${ }^{18}$ W. Seredyński, Rys dziejów wychowania $w$ biografiach i szkicach z szczególnym uwzględnieniem szkoły ludowej w Austrii i Polsce dla użytku seminariów nauczycielskich polskich, Tarnów 1891, p. 44; F. Majchrowicz, Historia pedagogii dla użytku seminariów nauczycielskich, Drohobycz 1901, p. 48.
} 
polacchi di storia delleducazione segnalano che non senza motivo le prime università italiane sorsero laddove si erano create circostanze favorevoli, quali un numero congruo di dotti professori e di giovani volonterosi e desiderosi di studiare, l'opportuno appoggio da parte delle autorità ecclesiastiche o laiche. La Scuola Medica Salernitana, la Scuola di Bologna, quelle di Perugia, Padova e Pisa ${ }^{19}$ ne sono l'esempio ideale. Gli studiosi polacchi di storia dell'educazione apprezzavano particolarmente il sistema universitario bolognese che servì da modello per altri atenei italiani ed europei, fra cui l'Accademia Cracoviana fondata nel 1364 da Casimiro il Grande, re di Polonia. ${ }^{20}$

Gli autori che trattano della storia dell'istruzione nell'Italia medievale sottolineano che nell'ambito della letteratura pedagogica dell'epoca si era sviluppato un particolare genere letterario dedicato all'educazione dei principi. Stanisław Kot afferma fra laltro che andava molto di voga la lettura del trattato di Egidio Colonna detto anche Egidio Romano De regimine principiam scritto agli inizi del sec. XIII e dedicato a Filippo il Bello, futuro re di Francia. I manuali polacchi di storia dell'educazione, trattando le opere pedagogiche italiane forniscono di solito una breve nota descrittiva e così fece anche $\mathrm{S}$. Kot presentando il trattato di Egidio Colonna: "l'autore italiano sottolinea come gravi sugli stessi genitori laici (ovviamente di ceti sociali superiori) l'obbligo di istruire ed educare i figli non soltanto alla fede, come succedeva fino ad allora, ma anche alla filosofia, etica, economia e politica e ritiene che anche alle femmine si addicano le occupazioni intellettuali. Sotto l'impulso vivificante del mondo classico l'ascetismo medievale diventa meno rigido, il culto della mortificazione carnale cede il posto all'indulgenza, e gli alimenti, il vestiario e lo svago dei fanciulli diventano oggetto di considerazione. Egidio consiglia ai giovani che si preparano alla vita politica di esercitarsi nel gioco della scherma e nei lavori manuali". ${ }^{21}$

Nei compendi di storia delleducazione si accenna anche a San Tommaso d'Aquino, filosofo e teologo napoletano, massimo rappresentante della scolastica medievale. I. Możdżeń rivolgendosi ai suoi lettori afferma che nonostante San Tommaso d'Aquino nella sua opera teologica e filosofica dedicasse poco spazio all'educazione, le sue idee in materia si distinguono per la loro originalità. Możdżeń ritiene tali le opinioni di San Tommaso sugli obiettivi dell'educazione,

\footnotetext{
19 S. Kot, Historia wychowania. Zarys podręcznikowy, 1924, p. 128.

${ }^{20}$ S. I. Możdżeń, Historia wychowania do 1795, ed. 2, Sandomierz 2006, p. 168.

${ }^{21}$ S. Kot, Historia wychowania. Zarys podręcznikowy, 1924, p. 136.
} 
sulla figura dell'educatore, sui metodi educativi, sulleducazione morale, religiosa, intellettuale e fisica. ${ }^{22}$

Il periodo della storia culturale che interessava maggiormente gli studiosi polacchi di storia dell'educazione è il Rinascimento. Dopo aver puntualmente sottolineato che l'Umanesimo e il Rinascimento erano nati in terra italiana, una terra ricca di monumenti artistici e storici dell'antica Roma e i cui abitanti si ritenevano eredi e successori dei Romani ${ }^{23}$ passavano a una analisi dettagliata dell'influsso dell'umanesimo sulla pedagogia. E rilevavano che in Italia le scuole dirette dal clero non godevano più della fiducia di un tempo e che le università di tradizione scolastica erano divenute oggetto di aspre critiche. Nellopinione degli studiosi polacchi l'epoca rinascimentale si caratterizzava fra l'altro per il fatto che le famiglie signorili e nobili nonché i ceti più abbienti affidavano leducazione dei propri figli a precettori privati, spesso umanisti di fama. Di carattere pionieristico fu l'attenzione data alleducazione fisica, in forte contrasto con il medioevale culto della mortificazione e il disinteresse per l'igiene del corpo. ${ }^{24}$

Nei manuali polacchi sull'argomento ampio spazio viene dedicato ad eminenti pedagogisti italiani, Pier Paolo Vergerio, Vittorino da Feltre, Maffeo Vegio e Enea Silvio Piccolomini. Ad avvicinare agli studenti polacchi le loro idee umanistiche sull'educazione fu il manuale di Stanisław Kot, profondo conoscitore dell'Umanesimo italiano, il quale considerava progressista il pensiero pedagogico italiano dell'epoca. L'interesse per le problematiche pedagogiche e didattiche si diffuse poi ancora di più con il rinvenimento, nel sec. XV, di testi pedagogici di Quintilio e Plutarco, il che diede ulteriore impulso a studi più approfonditi del sistema educativo dell'antichità e alla successiva fioritura della letteratura pedagogista. St. Kot era convinto che il pensiero pedagogico italiano fosse fortemente influenzato dalla tradizione classica e a titolo d'esempio riportava il più famoso trattato pedagogico quattrocentesco, De educatione liberorum et eorum claris moribus libri VI di Maffeo Vegio affermando che "si tratta ormai di una vera e propria pedagogia umanistica che tocca tutti i problemi connessi all'educazione". 25

22 S. I. Możdżeń, Historia wychowania do 1795, ed. 2, pp. 187-188.

23 S. Kot, Historia wychowania. Zarys podręcznikowy, 1924, p. 156.

24 S. Kot, Historia wychowania. Zarys podręcznikowy, 1924, p. 152.

${ }^{25}$ S. Kot, Historia wychowania. Zarys podręcznikowy, 1924, p. 154; vedi anche: F. Bizoń, Historia wychowania ze źródeł czerpana, dla użytku seminariów nauczycielskich, nauczycieli i ludzi zajmujących się wychowaniem, Lwów 1913, p. 67; F. Majchrowicz, Historia pedagogii dla użytku seminariów nauczycielskich, pp. 55-56. 
Della ricca letteratura pedagogica dell'umanesimo italiano gli autori polacchi dei manuali apprezzarono particolarmente il trattato di Enea Silvio Piccolomini futuro papa Pio II De liberorum educatione perché trasmetteva la conoscenza razionale circa l'educazione mentale e fisica dei principi diventando un modello per tanti altri autori di libri sullo stesso argomento. Che il trattato di Enea Silvio Piccolomini fosse uno di quelli che contribuirono a diffondere il pensiero pedagogico italiano negli altri paesi europei era un'opinione ampiamente condivisa. Si sottolineava poi il fatto che non solo la parola scritta ma anche altri fattori contribuirono alla divulgazione del pensiero pedagogico italiano. Infatti, i principi pratici dell'educazione umanistica venivano trasmessi alle altre nazioni. Gli storici osservano anche che un notevole impatto sulla diffusione del pensiero pedagogico umanistico ebbero il Concilio di Basilea (attraverso gli illustri umanisti della corte papale, i funzionari e i dignitari ecclesiastici di vari paesi ivi presenti appresero il culto per la letteratura antica) nonché l'invenzione della stampa.

Gli studiosi polacchi annotarono anche alcune manchevolezze del pensiero pedagogico umanistico. St. Kot osserva che "l'umanesimo non portò con sé tendenze democratiche. Ben lungi dall'estendere l'istruzione a tutti, si concentrava preferibilmente attorno alle corti dei nobili. Il patrimonio culturale antico che tanto stimava, non era facilmente accessibile a tutti: lo studio dellantichità richiedeva infatti tempo e notevole impegno finanziario. Un latino impeccabile non trovava poi pratica applicazione di massa: la pronunzia ciceroniana era utile solo ai ceti che in qualche modo partecipavano alla vita pubblica, facevano parte delle autorità statali o ecclesiastiche. L'educazione nello spirito umanistico gioco forza risultava quindi di carattere aristocratico" ${ }^{26}$ Comunque prevalevano opinioni positive; si poneva l'accento sul fatto che paragonato con il pensiero pedagogico italiano medievale quello dell'epoca rinascimentale era già progredito: non si limitava più a propagare l'istruzione fra il clero ma riconosceva la necessità di educare ed istruire anche la società laica.

Un argomento largamente trattato dagli storici polacchi delleducazione, nei loro scritti dedicati all'epoca rinascimentale, furono i viaggi dei giovani polacchi che si recavano nella Penisola Appenninica per studiare. L'Italia, culla della letteratura umanistica e dell'arte rinascimentale, fu nel secolo XVI la meta preferita dei futuri studenti. Si andava a studiare preferibilmente a Padova, che all'epoca teneva il primato nel campo dell'insegnamento di filologia, letteratura

${ }^{26}$ S. Kot, Historia wychowania. Zarys podręcznikowy, 1924, p. 158. 
e medicina, ma molti sceglievano anche l'ateneo bolognese. Nel sec. XVI fra gli studenti delle università italiane troviamo i nomi di eccellenti rappresentanti del mondo letterario e scientifico polacco: Niccolò Copernico, Klemens Janicki, Stanisław Hozjusz, Marcin Kromer, Stanisław Orzechowski, Jan Kochanowski, Andrzej Patrycy Nidecki, Łukasz Górnicki, Jan Zamojski, Mikołaj Sęp Sarzyński e Piotr Kochanowski. Gli storici ci tenevano a sottolineare che i polacchi si recavano in Italia non solo per studiare scienze; la permanenza in Italia permetteva loro di farsi una cultura, acquistare un certo garbo intellettuale e di urbanizzare i propri costumi, di conoscere la vita delle corti dei nobili italiani, di ammirare opere d'arte, di imparare la lingua italiana. ${ }^{27}$ Certo, non tutti erano tanto ambiziosi e non di rado succedeva che gli studenti invece di sfruttare al meglio il soggiorno italiano scialacquavano il denaro destinato alla loro educazione e fra un divertimento e l'altro cadevano nei vizi. E, una volta tornati in terra natia, addirittura ostentavano il loro disprezzo per la cultura e il sistema scolastico nazionali. ${ }^{28}$

Non poteva mancare nei manuali polacchi la dovuta attenzione al pensiero di Tommaso Campanella articolato nel trattato "La Città del Sole". Le sue idee utopistiche trovarono un riscontro positivo nel manuale pubblicato a cura di Łukasz Kurdybacha (all'epoca dell'indottrinamento politico): “Trattando leducazione dal punto di vista della richiesta sociale, non solo contemporanea ma anche quella futura, Campanella si rendeva perfettamente conto che per migliorare la condizione umana occorresse educare la gioventù alla vita in una società che non conosce più proprietà privata, sfruttamento né ingiustizia. Riteneva che il mezzo più adatto per educare i giovani al lavoro per il bene pubblico fosse l'insegnamento delle scienze naturali, la cui conoscenza e applicazione pratica avrebbero dovuto sicuramente contribuire all'aumento della produzione, e quindi a portare la prosperità e il benessere, a prevenire le malattie e a garantire le dovute cure. L'insegnamento delle scienze naturali basato sul metodo dimostrativo apriva nuovi larghi orizzonti all'insegnamento". ${ }^{29}$ Un giudizio meno positivo dei postulati pedagogici di Campanella è quello espresso da $S$. Litak che li ritiene radicali ed inapplicabili.

27 S. Kot, Historia wychowania. Zarys podręcznikowy, 1924, p. 195.

28 S. I. Możdżeń, Historia wychowania do 1795, ed. 2, p. 259.

${ }^{29}$ Historia wychowania, vol.1, a cura di Ł. Kurdybacha, Warszawa 1967, p. 476. 


\section{II pensiero pedagogico italiano nei secoli XVIII e XIX}

All'epoca dell'Illuminismo in Polonia si osserva un certo calo di interesse per il pensiero italiano nel settore culturale ed educativo. Lattenzione si rivolge verso le nuove idee pedagogiche illuministiche nate in Francia (gli scritti di Jean J. Rousseau) e in Inghilterra (John Locke). Il che non vuol dire che non ci si interessava dei progressi compiuti dagli Italiani in questo settore. Gli storici polacchi annotano l'impatto del pensiero pedagogico italiano sull'insegnamento settecentesco polacco e un indubbio contributo nel dare un nuovo impulso al sistema scolastico polacco da parte di coloro che avevano avuto la fortuna di studiare negli atenei italiani. Ne è illustre esempio don Stanisław Konarski, scolopio mandato a Roma per completare gli studi preso il Collegio Nazareno. Affascinato dal sistema scolastico italiano, Konarski volle riformare la scuola polacca seguendone la scia. ${ }^{30}$ Tornato in Polonia fondò nel 1740 il Collegium Nobilium, una scuola délite per figli dei magnati. Ai modelli italiani attinse anche Hugo Kołłątaj, riformatore dell’Accademia Cracoviana.

Scarso riflesso nei manuali polacchi trovò invece il pensiero pedagogico italiano dell'Ottocento. Un giudizio estremamente negativo del magistero italiano dellepoca lo troviamo in Chowanna (Larte delleducazione) di Bronisław Ferdynand Trentowski: "L'Italia, la Spagna e il Portogallo dal punto di vista della pedagogia sembrano un terreno lasciato da sempre incolto. Della gioventù si occupa la gerarchia oppure la cieca sorte. Il governo non se ne prende cura. Dapprima furono i gesuiti, poi i padri scolopi, oggi di nuovo sono i gesuiti a mantenervi il remoto, ormai debole riflesso della luce europea.". ${ }^{31}$ Ma tale eccessiva critica del pensiero pedagogico italiano è sicuramente in qualche misura ingiusta.

Comunque gli storici contemporanei dell'educazione e dell'istruzione giudicano positivamente lattività degli istituti religiosi italiani nel settore educativo, sottolineando che nell'ottocento proprio nella Penisola Appenninica si realizzò il rinnovamento religioso europeo e gli ordini dei gesuiti e dei dominicani ripresero la loro attività. I manuali polacchi accennano alla Congregazione delle Suore Passioniste di San Paolo della Croce, fondata a Firenze da Maria Maddalena Frescobaldi, che si occupava di istituti di correzione per le ragazze cadute

30 S. Kot, Historia wychowania. Zarys podręcznikowy, 1924, p. 314; S. Możdżeń, Historia wychowania do 1795, ediz. 2, p. 382.

${ }^{31}$ B. F. Trentowski, Chowanna czyli system pedagogiki narodowej jako umiejętności wychowania, nauki i oświaty, słowem wykształcenia naszej młodzieży, vol. 2, introduzione e commento di A. Walicki, Wrocław-Warszawa-Kraków 1979, p. 785. 
nella prostituzione. ${ }^{32} \mathrm{Ma}$ lo spazio maggiore è dedicato alla società salesiana fondata da San Giovanni Bosco e al suo ruolo nell'educazione della gioventù povera ed abbandonata. Il suo sistema di educazione preventiva divenne oggetto di studio di numerose analisi. ${ }^{33}$

\section{Il pensiero pedagogico italiano nei secoli XIX e XX}

Il Novecento dinamizza lo sviluppo della pedagogia e della didattica accelerando la nascita di nuovi orientamenti e modelli educativi. Fra i pedagogisti italiani che in modo creativo contribuirono ad arricchire il pensiero pedagogico gli storici polacchi apprezzano maggiormente la figura di Maria Montessori. Il manuale di S. Kot pubblicato nel 1934 riporta abbondanti informazioni su di lei. In Polonia, all'epoca della redazione del manuale, tra tutti gli scritti di Maria Montessori solamente la Casa dei bambini (Domy dziecięce, Varsavia 1913) era noto, grazie a una traduzione dal francese. L'autore polacco analizza tutti gli aspetti del sistema montessoriano rilevando che la pedagogista italiana rompe in modo radicale con i consueti metodi pedagogici applicati all'educazione dei bambini. S. Kot, in controtendenza rispetto alla posizione della maggior parte dei critici dellopera della Montessori, scorge alcune mancanze nel suo sistema. Osserva che la proposta montessoriana della pedagogia scientifica non dà i risultati attesi: è ancorata sostanzialmente alla fisiologia e alla psicologia e tralascia le finalità stesse dell'educazione. "Nel sistema montessoriano - argomenta poi S. Kot - ciò che suscita certe perplessità è il concetto di libertà e autonomia del bambino. Quest'ultimo viene a trovarsi in un ambiente del tutto artificioso, circondato da materiali didattici così organizzati che in pratica della sua libertà non rimane niente. Il sistema non permette lo sviluppo della fantasia, che la Montessori trascura. Quando il bambino vuole trattare il materiale didattico seguendo la propria fantasia, quando cioè invece di esercitarsi come previsto preferisce giocare, tale atto trasgressivo viene immediatamente interrotto; lo stesso vale per gli altri aspetti della fantasia: favole e disegno libero. Non vi è il gioco spontaneo attraverso il quale si esprime e sviluppa la personalità del bambino. Per tutti questi motivi, ed altri ancora, la pedagogia montessoriana, così apparentemente scientificamente studiata, oggi comincia a perdere la sua

32 S. I. Mozdżeń, Historia wychowania 1795-1918, ediz. 2, p. 38.

33 J. Draus, R. Terlecki, Historia wychowania. Wiek XIX i XX, pp. 63-64. 
popolarità a favore della corrente proposta da Froebel, il quale prescindendo dalla psicologia scientifica si rivolge direttamente all'anima del bambino nella sua interezza e sceglie il materiale da giocare a seconda e se in esso si rispecchia l'intero mondo del bambino". ${ }^{34}$

Nel periodo fra la prima e la seconda guerra mondiale in Polonia giungevano informazioni sulle riforme italiane, ma trovarono spazio solo nei manuali pubblicati dopo il 1945. Fra gli autori che offrono un quadro storico delleducazione italiana dopo il 1918 vi è Leonard Grochowski, coautore della Storia delleducazione, secolo XX (Historia wychowania wiek XX a cura di Józef Miąso. Lautore vi presenta gli inizi del sistema educativo pubblico, mettendo in rilievo il fatto che esso era nato nella seconda metà del sec. XIX, o meglio, nella fase finale dell'unificazione dell'Italia. Di seguito analizza le riforme del periodo interbellico realizzate da Giuseppe Lombardo-Radice, Benedetto Croce, Giovanni Gentile, dimostrando che le loro idee pedagogiche e le decisioni prese nel settore costituirono uno spunto per le trasformazioni del sistema scolastico ed educativo nello spirito fascista. A giudizio di L. Grochowski, le trasformazioni culturali e formative realizzate nel periodo a cavallo fra le due guerre mondiali furono fortemente influenzate dall'ideologia fascista "non furono che una più pericolosa variante della pedagogia capitalista, intrisa di idee nazionaliste e militariste, basata sullobbedienza cieca al Duce d'Italia (che ambiva di diventare trasmettitore dello spirito del Popolo), sulla disciplina e l'impegno ad appoggiare la dittatura come strumento dell'ordine sociale classista. Questi ideali educativi inculcati dalla scuola e dalle organizzazioni extrascolastiche depravavano le menti dei giovani italiani con risultati disastrosi. Ė del tutto comprensibile che con la caduta del governo fascista in seguito allo sfavorevole andamento della seconda guerra mondiale, tale pedagogia non solo perse il sostegno ufficiale ma venne rifiutata, come avvenne anche in Germania, in quanto negatrice della libertà e dell'umanitarismo". ${ }^{35}$ Gli storici dell'educazione polacchi sostengono che fu proprio la gioventù a subire in particolare le conseguenze dell'influenza distruttiva dell'ideologia fascista. ${ }^{36}$

${ }^{34}$ S. Kot, Historia wychowania. Zarys podręcznikowy, 1924, vol. 2. Wychowanie nowoczesne (od połowy w. XVIII do współczesnej doby, Lwów 1934, p. 339.

${ }^{35}$ L. Grochowski, Rozwój oświaty i wychowania we Włoszech do II wojny światowej, in: Historia wychowania wiek XX, a cura di J. Miąso, vol. 2, Warszawa 1980, p. 222.

36 S. I. Możdżeń, Historia wychowania 1918-1945, ediz. 2, p. 64. 
Gli studenti polacchi nei manuali utilizzati nello studio della materia hanno modo di trovare informazioni sulle riforme del sistema scolastico italiano del dopoguerra (la riforma del sistema nel 1962, e successiva modifica del 1990), nonché sull'insegnamento secondario superiore, il sistema universitario italiano, gli standard attuali e i programmi di studio. ${ }^{37}$

Indubbiamente si nota nei manuali polacchi di storia delleducazione quanto sia importante la conoscenza del pensiero pedagogico italiano. Particolare rilievo si dà all'eredità umanistica italiana mettendo in evidenza le numerose proposte pionieristiche del pensiero pedagogico. Ampio spazio va anche dedicato agli eminenti teorici e pratici dell'educazione, San Giovanni Bosco e Maria Montessori.

Oggi di crescente popolarità fra gli studenti polacchi godono i viaggi di studio in Italia che indubbiamente contribuiscono ad arricchire la conoscenza polacca della realtà italiana anche sul piano delle scienze pedagogiche.

Come deriva dall'articolo di cui sopra i manuali polacchi di storia dell'educazione contengono una ricca raccolta di informazioni sull'educazione e sul sistema scolastico in Italia nei secoli passati. La maggior parte degli autori sottolinea il fatto che le idee pedagogiche italiane erano pionieristiche, intrise dello spirito dell'umanesimo e nel periodo del tardo medioevo e del rinascimento hanno influenzato la formazione del pensiero educativo in altri paesi. Nei manuali non mancano le informazioni sui viaggi di studio dei Polacchi nelle università italiane, né il ritratto dei più illustri pedagogisti italiani. Nellera contemporanea, in un'epoca di sempre maggiore globalizzazione, è particolarmente indicato trattare, nei testi di studio umanistici, le questioni riguardanti la scienza e la cultura di altri stati. Ciò mostra le radici comuni dell'Europa, le tradizioni e gli ideali comuni, che hanno illuminato i nostri antenati e che sono presenti anche nel mondo contemporaneo.

\section{Bibliography}

Bartnicka K., Szybiak I., Zarys historii wychowania, Warszawa 2001.

Barycz H., Rozwoj historii oświaty, wychowania i kultury w Polsce, Kraków 1949.

Barycz H., Stanislaw Kot (1885-1975) - historyk polskiego Odrodzenia i Reformacji, “Biuletyn Biblioteki Jagiellońskiej”, vol. 28 (1978), nn. 1-2, pp. 5-22.

37 J. Draus, R. Terlecki, Historia wychowania. Wiek XIX i XX, p. 243. 
Bielska M., Historia pedagogii, Lwów 1890.

Bizoń F., Historia wychowania ze źródeł czerpana, dla użytku seminariów nauczycielskich, nauczycieli i ludzi zajmujących się wychowaniem, Lwów 1913.

Blaike J.S., Historia wychowania. Repetytorium przedegzaminowe, Lwów 1925.

Draus J., Terlecki R., Historia wychowania. Wiek XIX i XX, Kraków 2005.

Dybiec J., Stanislaw Kot (1885-1975), in: Złota ksiega Wydzialu Historycznego Uniwersytetu Jagiellońskiego, a cura di J. Dybiec, Kraków 2000, pp. 260-270.

Dybiec J., Recepcja idei pedagogicznych w Polsce, in: Recepcja w Polsce nowych kierunków i teorii naukowych, a cura di A. Strzałkowski, Kraków 2001, pp. 195-202.

Gadowski W., Z historii pedagogii. Czasy od początku chrześcijaństwa do końca średniowiecza, Kraków 1889.

Grochowski L., Rozwój oświaty i wychowania we Włoszech do II wojny światowej, in: Historia wychowania wiek XX, a cura di J. Miąso, vol. 2, Warszawa 1980.

Guz P., Zarys historii wychowania, parte 1. Opole 1961.

Historia wychowania w XX w. Dorobek i perspektywy, Kielce 1998.

Historia wychowania, vol. 1, a cura di Ł. Kurdybacha, Warszawa 1967.

Historia wychowania. Skrypt dla studentów studiów dziennych i zaocznych, a cura di J. Hellwig, Poznań 1994.

Kot S., Historia wychowania. Zarys podręcznikowy, Kraków 1924.

Krajewski M., dzieje wychowania i doktryn pedagogicznych, Płock 2003.

Krasuski J., Historia wychowania, Warszawa1985.

Kremer J., Podróż do Włoch, vol.1. Droga z Krakowa do Triestu. Opisanie Wenecji, in: Dzieła Józefa Kremera, vol. VI, Warszawa 1978.

Krolinski K., Zwięzły podręcznik historii pedagogii, Lwów 1907.

Majchrowicz F., Historia pedagogii dla użytku seminariów nauczycielskich, Drohobycz 1901.

Majorek C., System kształcenia nauczycieli szkół ludowych w Galicji doby autonomicznej (1971-1914). Wrocław 1971.

Marciniak Z., Zarys historii wychowania, Warszawa 1978.

Maver G., Podróże polskich pisarzy do Włoch, Rzym 1946.

Meissner A., Polskie podręczniki do historii wychowania, "Rozprawy z Dziejów Oświaty" vol. 32 (1989), pp. 155-167.

Michalski S., Miejsce historii wychowania w systemie nauk pedagogicznych, „Prace Komisji Pedagogiki i Psychologii Bydgoskiego Towarzystwa Naukowego”, Bydgoszcz 1981.

Możdżeń S. I., Historia wychowania do 1795, ed. 2, Sandomierz 2006.

Możdżeń S. I., Praca naukowa historyka oświaty, Sandomierz 1999.

Podgórska E., Kotłowski K., Materiały do historii wychowania, Łódź 1956.

Rutkowski S., Lisowski W., Doroba Z., Wybrane zagadnienia z dziejów wychowania w Polsce, Warszawa 1970. 
Rzepecki A., Skrót historii wychowania, Lwów 1921.

Seredyński W., Rys dziejów wychowania w biografiach i szkicach z szczególnym uwzględnieniem szkoły ludowej w Austrii i Polsce dla użytku seminariów nauczycielskich polskich, Tarnów 1891.

Sokolowski E., Skrót historii pedagogiki według najnowszych podręczników szkolnych, Warszawa 1927.

Szelejewski J., Historia wychowania w zarysie, Poznań 1962.

Trentowski B. F., Chowanna czyli system pedagogiki narodowej jako umiejętności wychowania, nauki i oświaty, słowem wykształcenia naszej młodzieży, vol. 2, introduzione e commento di A. Walicki, Wrocław-Warszawa-Kraków 1979.

Wołoszyn S., Dzieje wychowania i myśli pedagogicznej w zarysie, Warszawa 1964. 